\title{
Hydrogen Sorption on the Different Types of the Boron-Carbon Nanotubes
}

\author{
Sergei Boroznin ${ }^{1}$, Irina Zaporotskova ${ }^{1}$, Natalia Boroznina ${ }^{1}$, Lev Kozhitov ${ }^{2}$ \\ ${ }^{1}$ Volgograd State University \\ Prospect Universitetskii, 100, 400062, Volgograd, Russia \\ boroznina.natalya@volsu.ru; irinazaporotskova@gmail.com \\ ${ }^{2}$ NUST MISIS \\ Leninsky Prospekt, 4, 119049, Moscow, Russia \\ kozitov@misis.ru
}

\begin{abstract}
In this research interaction of atomic and molecular hydrogen with carbon nanotubes containing boron impurities is considered. Process was modelled by step-by-step approach of atom or molecule of hydrogen to a surface of a nanotube. Calculations were carried out with use of model of a molecular cluster within a method of density functional theory (DFT). The research of the electronic and energy structure of the complex received in a consequence of influence of step-by-step approach of adatom to a surface of a carbon nanotubes containing boron impurities was conducted.
\end{abstract}

Keywords: Boron-carbon nanotubes, Hydrogenation, Adsorption, Density functional theory, Quantum-chemical research.

(C) Copyright 2020 Authors - This is an Open Access article published under the Creative Commons Attribution License terms (http://creativecommons.org/licenses/by/3.0). Unrestricted use, distribution, and reproduction in any medium are permitted, provided the original work is properly cited.

\section{Introduction}

In-depth study of unique sorption and electronicpower properties carbon nanotubes caused search of the novel nanostructures [1]. In 2003 single-layer boroncarbon nanotubes were synthesized [2]. This matter is a new class of the nanotube systems with the unique physical and chemical properties offering many fields of their use in technology [3].

In the last decade, the number of a possible carbon nanotube (CNT) using as the materials capable to accumulate and store hydrogen that is the ideal environmentally friendly energy carrier widespread in the nature [3-6] is widely discussed. It will allow solving, in particular, a problem of storage and transportation of hydrogen. For these years, there are many researches devoted to theoretical and experimental study of sorption activity of carbon nanotubes concerning hydrogen $[7,8]$.

Moreover, it is possible to assume, that carbonboron $\mathrm{BC}$ nanotube will also have high sorption activity in relation to hydrogen. It caused high interest in fundamental studying mechanisms of interaction between hydrogen and born-carbon nanotubes. More detailed studying can define possibilities of their use during creation of new accumulators of hydrogen.

\section{Calculation Method}

This article describe the research of the mechanism of adsorption of hydrogen atom and molecule on an outer surface of boron-containing BC nanotube (6.6). Calculations have been carried out using quantum and chemical calculation methods of DFT (Density functional theory).

According to Density Functional Theory, the properties of a many-electron system including energy can be defined by using an electron density functional. The system is described by electronic density as $\rho(\mathrm{r})$ :

$$
\rho(r)=\int \ldots \int\left|\Phi_{e}\right|^{2} d \sigma_{1} d \sigma_{2} \ldots d \sigma_{N}
$$

where $\Phi$ e is the many-electron wave function of the system, $\sigma \mathrm{i}$ is the set of spin and spatial coordinates of electrons, $\mathrm{N}$ is the number of electrons. Thus, $\rho(\mathrm{r})$ is a function of only three spatial coordinates $r$ of the point 
at which $\rho(\mathrm{r})$ gives the probability of detecting any of the electrons of the molecule [9].

If any property of the ground state of a molecule can be expressed in terms of $\rho$, then the electron energy in the DFT is:

$$
E[\rho]=T[\rho]+V_{e n}[\rho]+V_{e e}[\rho]
$$

where $T[\rho]$ is the kinetic energy, $V_{\text {en }}[\rho]$ is the potential energy of electron-nuclear interactions, $\mathrm{V}_{\text {ee }}[\rho]$ is the energy of electron-electron interactions, which can be written as:

$$
\begin{aligned}
& V_{e e}[\rho]=V_{\text {Coul }}[\rho]+V_{x c}[\rho] \\
& \mathrm{V}_{\text {ee }}[\rho]=\mathrm{V}_{\text {Coul }}[\rho]+\mathrm{V}_{\mathrm{xc}}[\rho]
\end{aligned}
$$

where $V_{\text {Coul }}[\rho]$ is the energy of the Coulomb interaction of electrons, and $\operatorname{Vxc}[\rho]$ is the exchangecorrelation energy.

The functionals $\mathrm{T}[\rho], \mathrm{V}_{\mathrm{en}}[\rho]$ and $\mathrm{V}_{\text {Coul }}[\rho]$ can be found exactly [9]. For the exchange-correlation potential $\operatorname{Vxc}[\rho]$, the exact representation is not known and there are a large number of models for its description. DFT is used with various functionals and one of the most popular is B3LYP, a hybrid functional that includes three components of the exchange functional (exact HartreeFock exchange operator, Becke functional and Slater functional, and the correlation part is a combination of the Lee-Yang-Parr functional (LYP) and Vosko-VilkaNusar ( VWN). A feature of this approach is that the three exchange components are taken with weighting factors selected based on comparison with experimental data. As a result, the approach takes on the characteristics of a semi-empirical method. It turns out that its accuracy in most cases is significantly higher than in the case of methodologically "pure" functionals. Apparently, this is a consequence of the fact that the exchange energy is nonlocal in nature and any attempts to reduce it to local functionals lead to errors. The Hartree-Fock exchange makes it possible to consider this nonlocality. Therefore, in the presented theoretical study, the B3LYP functional was used within the framework of the density functional theory. For the B3LYP hybrid functional, good convergence of experimental and theoretical results for carbon systems has been proved. The error of theoretical calculations for them is no more than $1 \%$ in terms of geometrical parameters (interatomic bonds and bond angles) and total energies.

\section{Formulation of the problem}

The possibility of hydrogen adsorption on an outer surface of one-wall BC5 nanotube is investigated in this research. A typical cluster of this nanotube is shown in fig. 1.

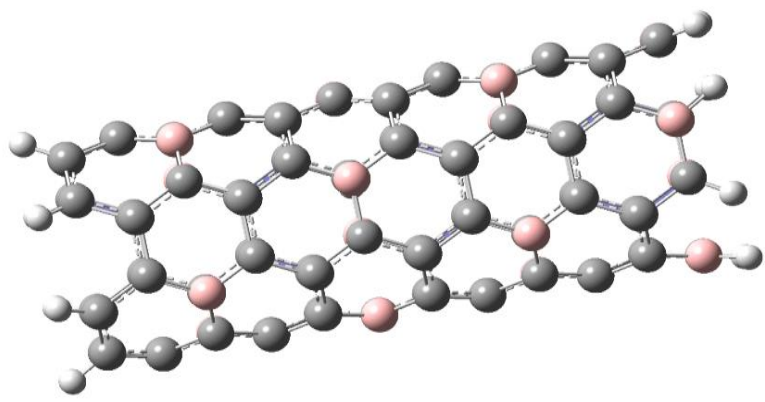

Figure 1 . The extended elementary cell of BC5 nanotubes $(6,6)$ of mutual orientation of $\mathrm{C}$ and $\mathrm{B}$ atoms.

The interaction between BCNT and $\mathrm{H}$ atom has been modeled using the step-by-step approaching to the surface of the nanotube (with a step $0,1 \mathrm{~A}$ ). The hydrogen atom located straight above the sorption center in all cases. The DFT calculation suggested us to build the potential energy curves (fig. 2). The view of these curves allowed us to make the conclusion that atomic adsorption is possible for all variants (fig. $2 \mathrm{a}$ and $2 \mathrm{~b}$ ). However, hydrogen molecule doesn't have any kind of interaction with nanotube surface (fig. 2c and 2d). It could be explained with high force of interatomic interaction in the hydrogen molecule.

The analysis of the electronic structure showed that the positive charge is located on the hydrogen atom while the boron or carbon atom of the nanotube surface get the negative one. This means that along with the weak van der Waals interaction Coulomb interaction arises between a boron (or carbon) atom and a hydrogen atom.

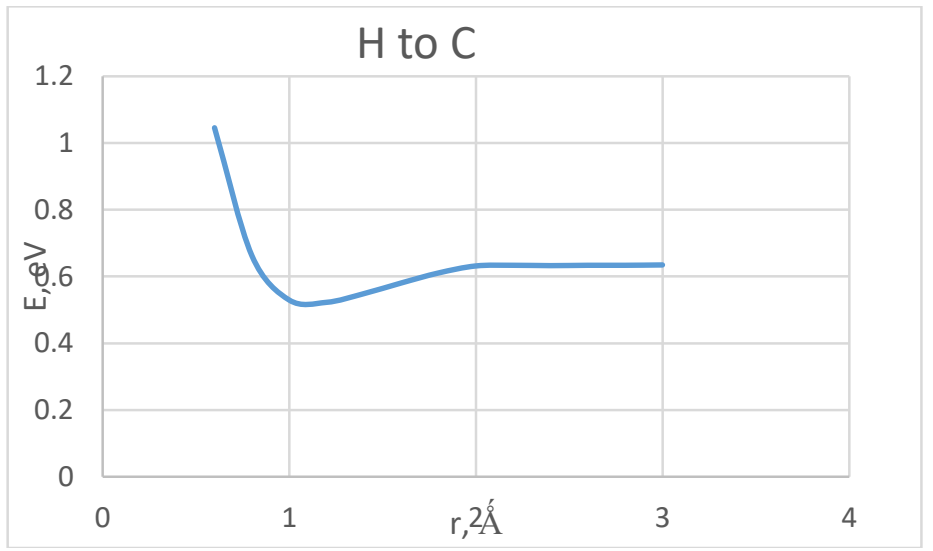

a) 


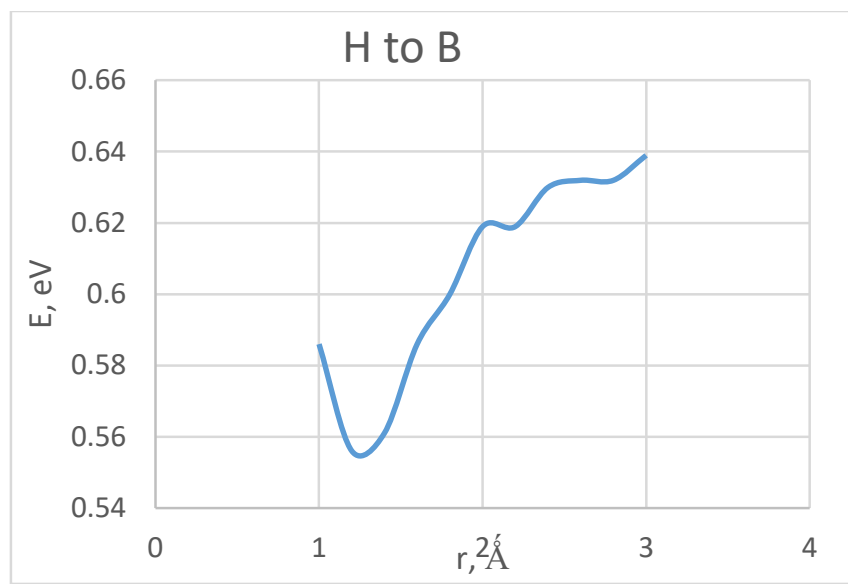

b)

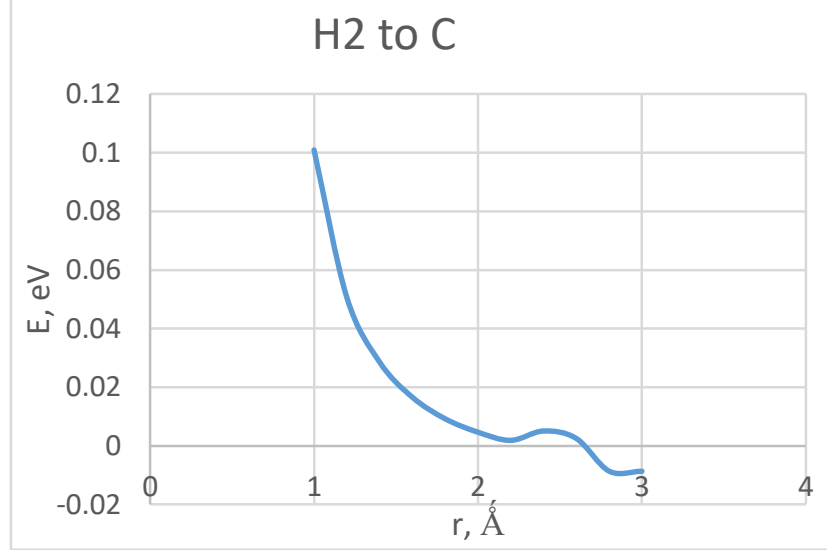

c)

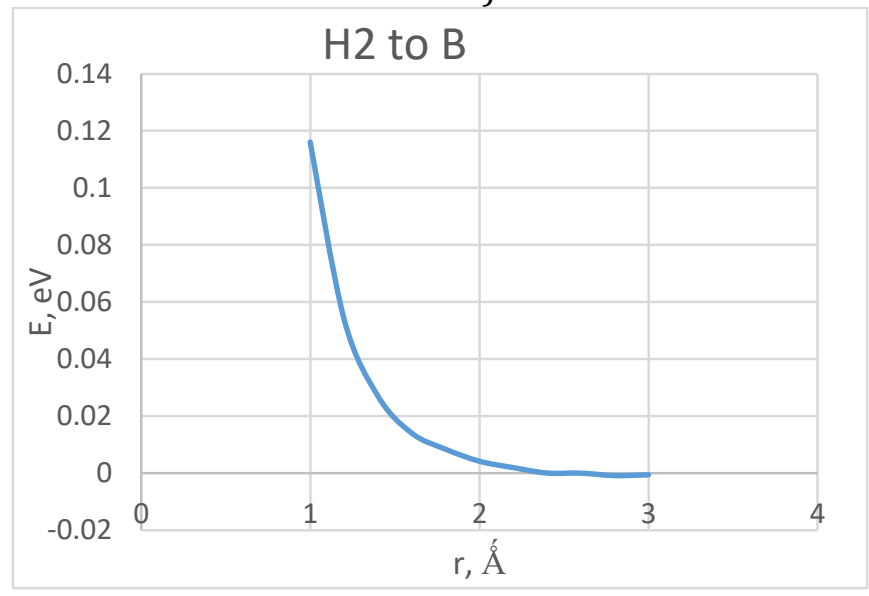

d)

Figure 2. The energy curves for the adsorption process of an

$\mathrm{H}$ atom and molecule on the external surface of BC5 nanotubes: a), b) above a boron/carbon atom for hydrogen atom; c), d) above a boron/carbon atom for hydrogen molecule.

The question remained open: does the concentration of boron atoms near the sorption center affect the course of adsorption processes? To respond to it, the results were compared with previously obtained data for the BC3 of nanotubes of types A and B $[10,11]$. Hydrogen was located at 4 positions above the surface of the nanotube (Figure 3). Computer simulation of adsorption was carried out in a similar manner to the previous study.

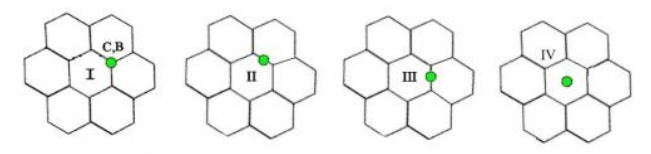

Figure 3. Location of hydrogen atom above the surface of nanotube.

Based on the results of the study, the energy curves of the atom's interaction with the BCNT surface were built (Fig. 4-6). The main parameters of the study are given in Table 1 and Table 2.

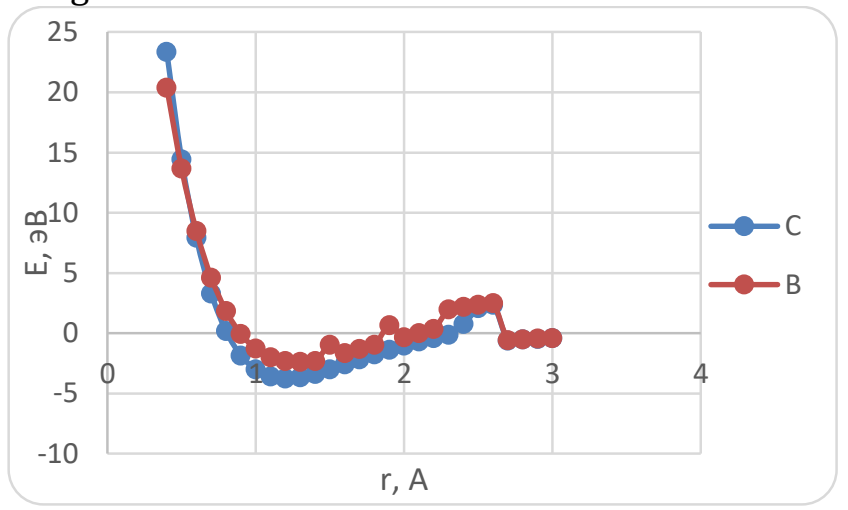

a)

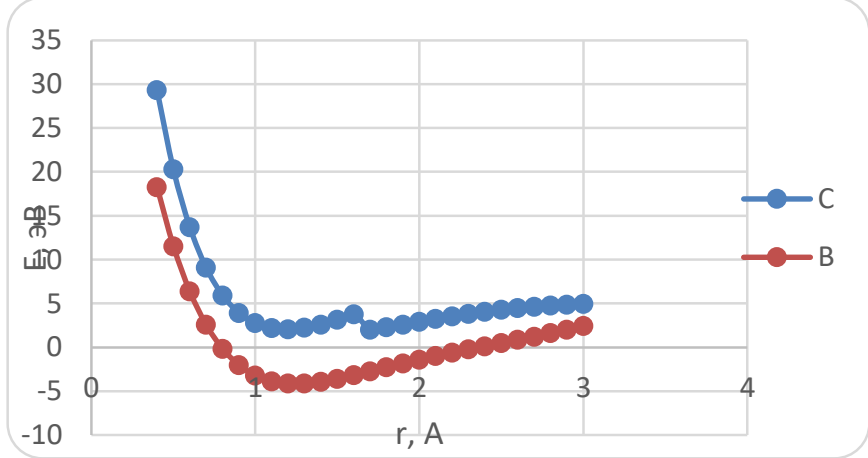

b)

Figure 4. The potential energy profile of the adsorption process of a hydrogen atom on the surface of $\mathrm{BC} 3$ nanotube for variant I: a) BC3 nanotube of type A; b) BC3 type B nanotube. 


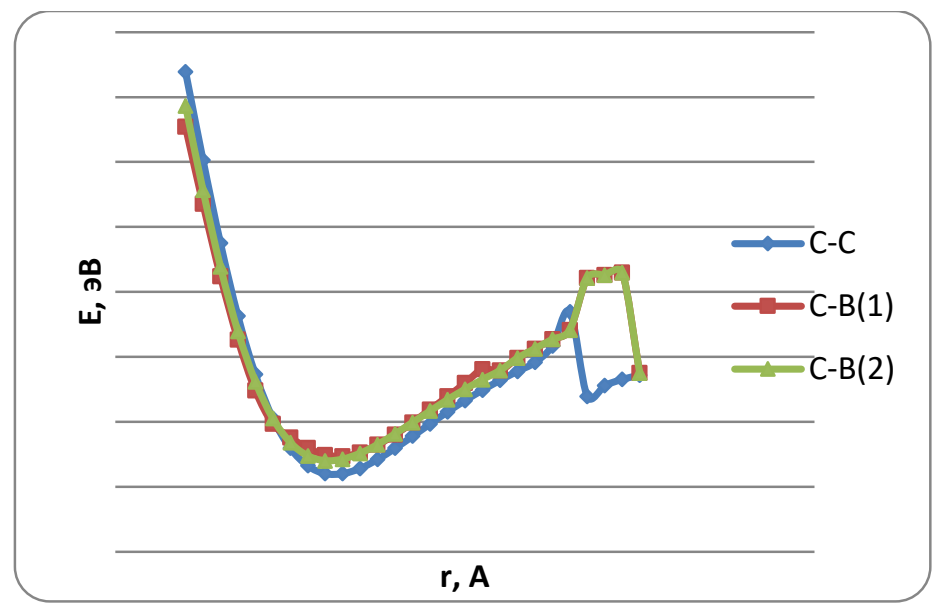

a)

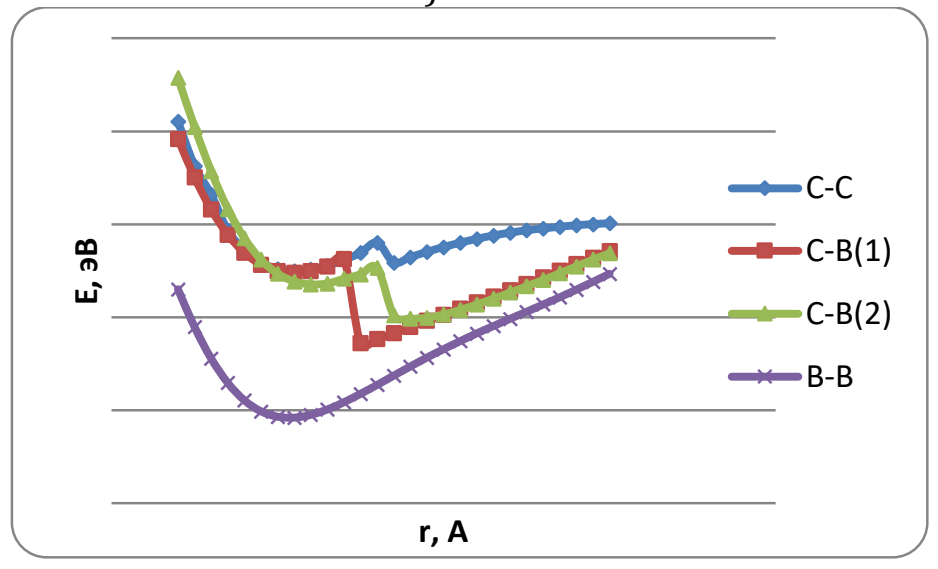

b)

Figure 5. The potential energy profile of the adsorption process of a hydrogen atom on the surface of $\mathrm{BC} 3$ nanotubes for variant II: a) BC3 nanotube of type A; b) BC3 type B nanotube.

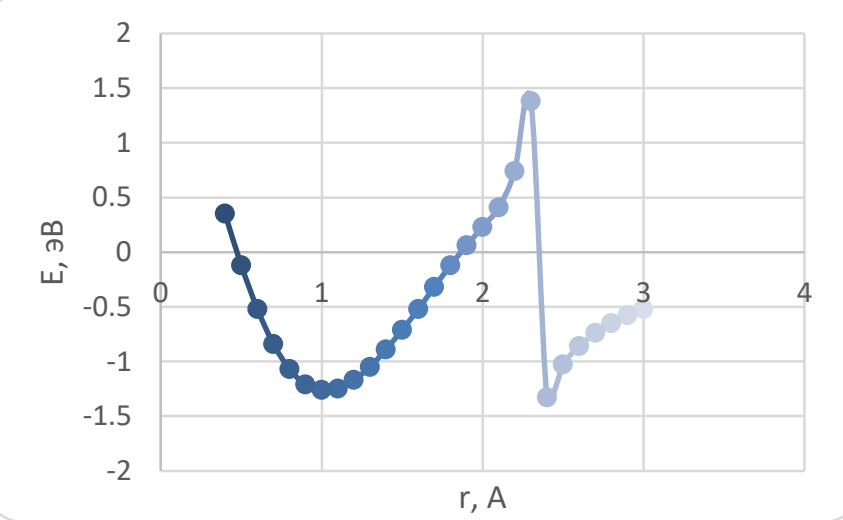

a)

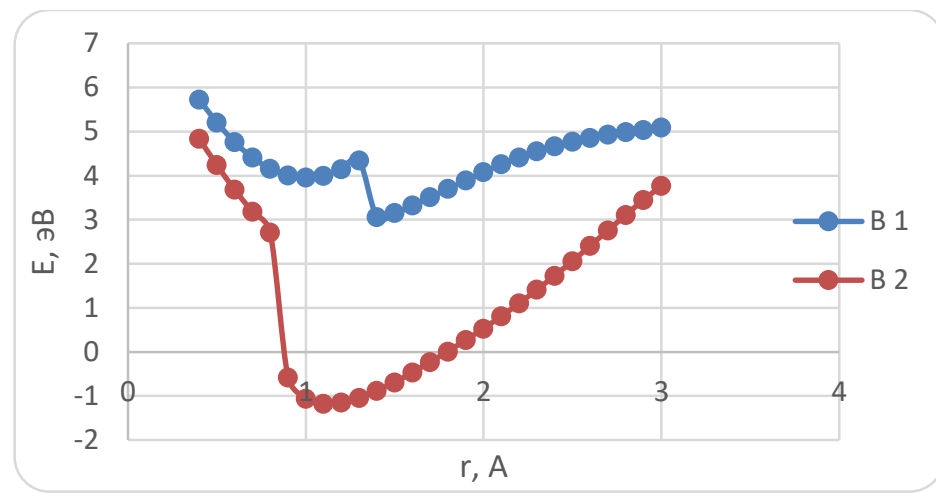

b)

Figure 6. The potential energy profile of the hydrogen atom adsorption process on the surface of $\mathrm{BC} 3$ nanotube for variant IV a) BC3 nanotube A type; b) BC3 nanotube of type B, a curve with the designation B1- above the centre of a hexagon with one boron atom, B2- with two boron atoms

Table 1. The main characteristics of atomic hydrogen adsorption processes on the surface of borocarbon nanotubes of type A for the considered options for the orientation of atom $\mathrm{H}$ relative to the surface of tubulens: Ead - adsorption energy; rad - adsorption distance; QA are effective charges on adatoms.

\begin{tabular}{|l|l|l|l|}
\hline & $\mathrm{R}_{\mathrm{ad}}, \hat{\AA}$ & $\mathrm{E}, \mathrm{eV}$ & $\mathrm{Q}_{\mathrm{A}}$ \\
\hline Above B atom & 1,3 & 1,09 & 0,20 \\
\hline Above C atom & 1,2 & 2,45 & 0,22 \\
\hline Above C-C bond & 1,3 & 1,09 & 0,30 \\
\hline Above C-B(1) bond & 1,3 & 0,80 & 0,26 \\
\hline Above C-B(2) bond & 1,2 & 1,63 & 0,23 \\
\hline $\begin{array}{l}\text { Above the hexago } \\
\text { centre }\end{array}$ & 1,0 & 1,02 & 0,61 \\
\hline
\end{tabular}

Table 2. The main characteristics of atomic hydrogen adsorption processes on the surface of borocarbon nanotubes of type B for the considered options for orientation of atom $\mathrm{H}$ relative to the surface of tubulens: Ead - adsorption energy; rad - adsorption distance; QA - effective charges on adatoms

\begin{tabular}{|c|c|c|c|}
\hline & $\mathrm{R}_{\mathrm{ad}}, \AA \dot{A}$ & $\mathrm{E}, \mathrm{eV}$ & $Q_{A}$ \\
\hline Above $\mathrm{B}$ atom & 1,2 & 1,90 & $-0,14$ \\
\hline Above $\mathrm{C}$ atom & 1,2 & 0,04 & 0,21 \\
\hline Above $\mathrm{C}-\mathrm{C}$ bond & 1,0 & 0,04 & 0,20 \\
\hline Above C-B(1) bond & 1,5 & 0,06 & 0,08 \\
\hline Above C-B(2) bond & 1,8 & 0,11 & 0,12 \\
\hline Above B-B bond & 1,1 & 0,16 & 0,05 \\
\hline $\begin{array}{l}\text { Above the center of a } \\
\text { hexagon with one boron } \\
\text { atom }\end{array}$ & 1,4 & 0,03 & 0,23 \\
\hline
\end{tabular}




\begin{tabular}{|l|l|l|l|}
\hline $\begin{array}{l}\text { Above the center of a } \\
\text { hexagon with two boron } \\
\text { atoms }\end{array}$ & 1,1 & 0,05 & $-0,19$ \\
\hline
\end{tabular}

\section{Conclusion}

Thus, theoretical studies have shown that the described borocarbon nanotubes interact with atomic hydrogen and do not react to the presence of a $\mathrm{H} 2$ molecule. The $\mathrm{H}$ atom is attached to the nanotubes at a distance corresponding to the chemical bond between the elements. Values of adsorption energy make it possible to predict formation of metastable complex "hydrogen atom - borocarbon nanotube." Molecular hydrogen does not react with the nanotube. The reason for this may be a strong bond between hydrogen atoms, preventing them from interacting with other substances without the application of external forces. The analysis of the electronic structure showed that the positive charge is located on the hydrogen atom while the boron or carbon atom of the nanotube surface get the negative one. This means that along with the weak van der Waals interaction Coulomb interaction arises between a boron (or carbon) atom and a hydrogen atom. Studies have shown that boron-carbon nanotubes can act as storage facilities for atomic hydrogen, while allowing it to be separated from molecular, due to its sorption capacity. For boron-containing (BC3) nanotubes, when the adsorption center is located above boron atoms, in the center of a hexagon containing two B atoms, and above the B-B bond center, the adsorption process of atomic hydrogen is energetically most favorable, as evidenced by the adsorption energy values and surface profiles of the potential energies of these processes. The adsorption energy of the $\mathrm{H}$ atom for borocarbon and boronitride nanotubulenes is approximately the same and is $\approx 1,5 \mathrm{eV}$ [12], while for pure boron nanotubes it is small $(0.07 \mathrm{eV})$ [13]. The best adsorbent of atomic hydrogen on the outer surface are carbon nanotubes, since their adsorption energy exceeds these values for other types of nanotubes $(4.07 \mathrm{eV})$ [1]. Therefore, it is possible to build a number of activities of some types of tubulenes in relation to atomic hydrogen (from more activity to less): carbon, boron-containing (BC3 and BN), boron hexagonal NTs.

\section{Acknowledgements}

The reported research was funded by Russian President's grant № 798.2019.1 and Russian President's grant № MK-1758.2020.8.

\section{References}

[1] I. V. Zaporotskova, "Carbon and Uncarbon nanomaterials and composite structures on their base: structure and electronic properties," Volgograd State University Publishing, Volgograd, 2009.

[2] G. G. Fuentes, E. Borowiak-Palen, M. Knupfer, T. Pichler, J. Fink, L. Wirtz, A. Rubio, "Formation and electronic properties of BC3 single-wall nanotubes upon boron substitution of carbon nanotubes," Phys. Rev. B., vol. 69, p. 245403, 2004.

[3] A. V. Yeletsky, "Sorption properties of carbon nanostructures," Advances in Physical Sciences, vol. 47, pp. 1119-1154, 2004.

[4] U. S. Nechaev, O. K. Alexeeva, "Methodological, applied and thermodynamic aspects of hydrogen sorption by graphite and related carbon nanostructures," Russian Chemical Reviews, vol. 73, no. 12, pp. 1211-1238, 2004.

[5] I. V. Zaporotskova, S. V. Boroznin, E. V. Perevalova, "Investigation of oxidation in boron-containing nanotubes," Nanosci. Nanotechnol. Lett, vol. 4, pp. 1096-1099, 2012.

[6] N. G. Lebedev, I. V. Zaporotskova, L. A. Chernozatonskii, "Fluoration of carbon nanotubes within molecular cluster method," International journal of quantum chemistry, vol. 96, no. 2, pp. 149-154, 2004..

[7] A. Nikitin, H. Ogasawara, D. Mann, R. Denecke, Z. Zhang, H. Dai, K. Cho, A. Nilsson, "Hydrogenation of single-walled carbon nanotubes," Phys. Rev. Lett, vol. 95, p. 225507, 2005.

[8] M. J. S. Dewar, W. Thiel, "Ground states of molecules. The MNDO method. Approximations and Parameters," J. Amer. Chem. Soc, vol. 99, pp. 4899-4906, 1977..

[9] W. Koch, M. Holthausen, "Chemist's Guide to Density Functional Theory," Weinheim: Wiley-VCH, pp. 19-28, 2002.

[10] I.V. Zaporotskova, E.V. Perevalova, D.I. Polikarpov, S.V. Boroznin "Electronic structure and characteristics of some types of boron-containing nanotrubs" in Bulletin of Volgograd State University. Section 10: Innovation, No. 6, pp. 81-86, 2012.

[11] S. V.Boroznin, I. V. Zaporotskova, E.V. Boroznina, D.I. Polikarpov "Adsorption of atomic hydrogen on the surface of the boron-carbon nanotubes" 
Russian Journal of General Chemistry, vol. 83, no. 8, pp.1580-1585, 2013.

[12] N.G. Lebedev, I.V. Zaporotskova, L.A. Chernozatonsky "Quantum chemical analysis of the interaction of a hydrogen atom with boronitride nanotubes" Chemical physics, vol. 25, no. 7, pp. 101-111, 2006.

[13] E.V. Perevalova, S.V. Boroznin, I.V. Zaporotskova, A.Yu. Stepanova "Adsorption of light atoms on the surface of boron nanotrubs" Metal technology, no. 10 , pp. $25-29.2010$. 